\title{
Exact Topological Soliton Solutions to the Strongly Perturbed Family of Sine-Gordon Type Equations
}

\author{
Stephen Johnson, Essaid Zerrad, Sokratis Makrogiannis, Jun Ren \\ Division of Physics, Engineering, Mathematics, and Computer Science Delaware State University Dover, DE, USA \\ Email: ezerrad@desu.edu
}

How to cite this paper: Johnson, S., Zerrad, E., Makrogiannis, S. and Ren, J. (2019) Exact Topological Soliton Solutions to the Strongly Perturbed Family of Sine-Gordon Type Equations. Journal of Applied Mathematics and Physics, 7, 2401-2422 https://doi.org/10.4236/jamp.2019.710163

Received: September 9, 2019

Accepted: October 19, 2019

Published: October 22, 2019

Copyright $\odot 2019$ by author(s) and Scientific Research Publishing Inc. This work is licensed under the Creative Commons Attribution International License (CC BY 4.0).

http://creativecommons.org/licenses/by/4.0/

\begin{abstract}
This paper uses the Ansatz method to solve for exact topological soliton solutions to sine-Gordon type equations. Single, double, and triple sine-Gordon and sine-cosine-Gordon equations are investigated along with dispersive and highly dispersive variations. After these solutions are found, strong perturbations are added to each equation and the new solutions are found. In solving both the perturbed and unperturbed sine-Gordon type equations, constraints are imposed on the parameters. The novel contributions of the authors are the soliton solutions to the strongly perturbed sine-Gordon equation and its variations. These results are important to the study of Josephson junctions, crystal dislocations, ultra-short optical pulses, relativistic field theory, and elementary particles.
\end{abstract}

\section{Keywords}

Topological Soliton, Kink Soliton, Sine-Gordon Equation, Dispersion, Perturbation

\section{Introduction}

The sine-Gordon equation (SGE) has applications to Josephson junctions, crystal dislocations, ultra-short optical pulses, relativistic field theory, and elementary particles [1].

A Josephson junction is a pair of superconductors separated by a thin material that is not superconducting. Brian Josephson predicted that a pair of superconducting electrons could tunnel through the non-superconducting material. Josephson junctions can be used in computer circuitry, increasing the speed of computations. They can also be used in Superconducting Quantum Interfering 
Devices, or SQUIDs. SQUIDs can measure minute changes in voltages and magnetic fields. Because of this, SQUIDs can be used to measure neural activity in the brain, heart activity [2], and even submarine detection [3].

A crystal dislocation is an irregularity within the crystal structure. There are two types of dislocations. An edge dislocation occurs when one plane of atoms only extends half-way through the crystal. This causes the planes to bend around it. A screw dislocation looks like the Riemann surface of the complex logarithm. Dislocations can also be a combination of the two types. Studying these dislocations can help material scientists improve the strength of metals. The kink solitons of the SGE can be used to model the interactions of these dislocations [4].

Ultrashort pulses allow scientists to study ultrashort processes and allow for optical data transmission. Some ultrashort processes of interest include electron dynamics within semiconductors, light-induced phase changes of metals, plasma dynamics, and chemical reactions [5]. Light pulses can be used to transmit data at very high rates due to high optical frequencies [6].

In relativistic field theory, topological solitons, or kinks, can be used to model undiscovered particles such as magnetic monopoles and cosmic strings [7].

The goal of this paper is to find exact solutions to strongly perturbed sine-Gordon (SG) type equations. Recent works in the literature proposed analytical and numerical solutions to this problem [8] [9] [10] [11]. In this work, we find exact solutions by means of the Ansatz method. This research is a direct continuation of the research done in [12] [13] [14] [15] and [16]. The research done in those papers was primarily to find solutions to the sine-Gordon equation and its variations under small, adiabatic perturbations. The solutions found in this paper are for strong perturbations to those same equations. In Section 2 we describe the Ansatz method, and in Section 3 we propose exact soliton solutions to sine-Gordon type equations and higher order dispersive versions. We conclude this work with a summary of our methods, the applicability of our results, and possible avenues of future work.

\section{The Ansatz Method}

The typical nonlinear wave equation that will be studied in this dissertation is of the form

$$
q_{t t}-q_{x x}+L(q)+N(q)=0
$$

where $x$ and $t$ represent partial derivatives with respect to space and time respectively, and where $L(q)$ represents the linear terms, including derivatives of $q$, and $N(q)$ represents the nonlinear terms, including derivatives of $q$. The Ansatz method requires a guess for the solution. Call this guess the particular solution $\hat{q}(x, t)$. Once an initial guess is made for $\hat{q}(x, t)$, that solution is put into the original nonlinear wave Equation (1)

$$
\hat{q}_{t t}-\hat{q}_{x x}+L(\hat{q})+N(\hat{q})=0
$$

The above equation is then simplified down to a sum of linearly independent 
functions,

$$
0=\sum_{i=0}^{n} c_{i} F_{i}(x, t)
$$

where $c_{i}$ are the constant coefficients of the linearly independent functions $F_{i}(x, t)$. In order to satisfy (2), it is necessary to set all coefficients equal to zero

$$
c_{i}=0 \quad \forall i \in\{0,1,2, \cdots, n\}
$$

In completing this step, one of two things will happen. If a critical parameter must be set to zero, then the particular solution $\hat{q}(x, t)$ has been proven to be invalid. Otherwise, this is proof that $\hat{q}(x, t)$ is a particular solution to (1). In the latter case, setting the $c_{i}$ to zero will lead to some constraints on the parameters of (1) and the internal parameters of $\hat{q}(x, t)$.

\section{Soliton Solutions}

\subsection{Sine-Gordon Equation and Its Type}

This first subsection will study the SGE and other similar equations without higher order dispersion terms. The same equations with the addition of fourth-order and sixth-order dispersion will be studied in the subsections thereafter.

\subsubsection{Sine-Gordon Equation}

The SGE studied in this paper is

$$
q_{t t}-k^{2} q_{x x}+a \sin q=0
$$

The kink Ansatz that will be used to solve this equation, given by [12], is

$$
q(x, t)=4 \arctan \{A \exp [B(x-v t)]\}
$$

The variable $E$ will be used henceforth, where $E=A \exp [B(x-v t)]$. Inserting (4) into (3) yields

$$
4 \frac{E-E^{3}}{\left(1+E^{2}\right)^{2}}\left[B^{2}\left(v^{2}-k^{2}\right)+a\right]=0
$$

Solving for $B$ gives

$$
B= \pm \sqrt{\frac{a}{k^{2}-v^{2}}}
$$

It turns out that $A= \pm \mathrm{e}^{-B x_{0}}$ represents the starting location of the soliton at $x_{0}$. Positive $A$ represents a bright soliton whereas negative $A$ represents a dark soliton. The sign of $A \cdot B$ determines the direction of the internal twist in the kink. These things hold true whenever the solution structure is an arctangent of an exponential.

The bright solution to the unperturbed SGE is

$$
q(x, t)=4 \arctan \left\{\exp \left[ \pm \sqrt{\frac{a}{k^{2}-v^{2}}}\left(x-x_{0}-v t\right)\right]\right\}
$$


The result above is already well known [1] [12] [13] [16] [17] [18]. Now that we have the exact solution to the unperturbed SGE, we will use the same Ansatz to find the exact solution to the strongly perturbed SGE. The variable $R$ will be used to hold all of the perturbation terms, where

$$
R=\beta q_{t}+\gamma q_{x}+\delta q_{x t}+\lambda q_{t t}+\sigma q_{x x t}+v q_{x x x x}
$$

In Josephson junctions, $\beta$ represents the dissipative losses of electrons tunneling across a dielectric barrier, $\gamma$ comes about from an inhomogeneous part of the local inductance, $\delta$ accounts for the diffusion, $\lambda$ results from an inhomogeneity of the capacitance, $\sigma$ arises due to current losses along the barrier, and $v$ contains the higher order spatial dispersion [18].

The perturbed SGE is thus

$$
q_{t t}-k^{2} q_{x x}+a \sin q=R
$$

Using the same Ansatz from (4), and performing the calculations found in Appendix A, we find the following relationships:

$$
\begin{aligned}
& \gamma=\beta \nu \\
& \sigma=0 \\
& v=0
\end{aligned}
$$

The bright soliton solution to the perturbed SGE is

$$
q(x, t)=4 \arctan \left\{\exp \left[ \pm \sqrt{\frac{a}{k^{2}-\delta v-(1-\lambda) v^{2}}}\left(x-x_{0}-v t\right)\right]\right\}
$$

with the constraints found in (6). These results have already been reported in [12], and are reproduced here in order to show the method that will be used henceforth.

\subsubsection{Sine-Cosine-Gordon Equation}

The sine-cosine-Gordon (SCGE) equation is

$$
q_{t t}-k^{2} q_{x x}+a \sin q+b \cos q=0
$$

The kink Ansatz for this equation is

$$
q(x, t)=4 \arctan \{A \exp [B(x-v t)]\}+C
$$

The solution to the unperturbed SCGE is

$$
q(x, t)=4 \arctan \left\{\exp \left[ \pm \frac{\left(a^{2}+b^{2}\right)^{\frac{1}{4}}}{\sqrt{k^{2}-v^{2}}}\left(x-x_{0}-v t\right)\right]\right\}-\arctan \left(\frac{b}{a}\right)
$$

The perturbed SCGE is

$$
q_{t t}-k^{2} q_{x x}+a \sin q+b \cos q=R
$$

where $R$ again contains all of the perturbation terms from (5). Using the same Ansatz from (7), and setting the coefficients of the linearly independent functions to zero as is done in Appendix A leads to the same matrix equation. The solution to the perturbed SCGE is 


$$
q(x, t)=4 \arctan \left\{\exp \left[ \pm \frac{\left(a^{2}+b^{2}\right)^{\frac{1}{4}}}{\sqrt{k^{2}-\delta v-(1-\lambda) v^{2}}}\left(x-x_{0}-v t\right)\right]\right\}-\arctan \left(\frac{b}{a}\right)
$$

\subsubsection{Double Sine-Gordon Equation}

The double sine-Gordon equation (DSGE) is

$$
q_{t t}-k^{2} q_{x x}+a_{1} \sin q+a_{2} \sin 2 q=0
$$

The first Ansatz we will investigate is

$$
q(x, t)=2 \arctan \{A \sinh [B(x-v t)]\}
$$

Substituting (9) into (8) and setting the coefficients to the linearly independent functions both to zero gives us the first solution to the unperturbed DSGE. The details of this process are given in Appendix B.

$$
q(x, t)=2 \arctan \left\{\sqrt{\frac{a_{1}}{a_{1}-2 a_{2}}} \sinh \left[ \pm \sqrt{\frac{2 a_{2}-a_{1}}{k^{2}-v^{2}}}(x-v t)\right]\right\}
$$

For the soliton to exist, it is necessary that $2 a_{2}>a_{1}$ and $a_{1}<0$.

The second Ansatz for (8), found in [13], is

$$
q(x, t)=2 \arctan \{A \tanh [B(x-v t)]\}
$$

The resulting equation from combining (8) and (10) and setting the coefficients of the linearly independent functions to zero leads to the second solution to the unperturbed DSGE. Again, the details of this process are given in Appendix B.

$$
q(x, t)=2 \arctan \left\{\sqrt{\frac{a_{2}+a_{1}}{a_{2}-a_{1}}} \tanh \left[ \pm \frac{1}{2} \sqrt{\frac{a_{2}^{2}-a_{1}^{2}}{a_{2}\left(k^{2}-v^{2}\right)}}(x-v t)\right]\right\}
$$

where $a_{2}>\left|a_{1}\right|$.

The perturbed DSGE is

$$
q_{t t}-k^{2} q_{x x}+a_{1} \sin q+a_{2} \sin 2 q=R
$$

Here, we will use the first Ansatz, (9). Solving as before leads to the first solution to the perturbed DSGE. This process is described in Appendix B.

$$
q(x, t)=2 \arctan \left\{\sqrt{\frac{a_{1}}{a_{1}-2 a_{2}}} \sinh \left[ \pm \sqrt{\frac{2 a_{2}-a_{1}}{k^{2}-\delta v-(1-\lambda) v^{2}}}(x-v t)\right]\right\}
$$

This requires the constraints found in (6) and $2 a_{2}>a_{1}$ and $a_{1}<0$.

Using the second Ansatz, (10), we get the second solution to the perturbed DSGE. This process is also described in Appendix B.

$$
q(x, t)= \pm 2 \arctan \left\{\sqrt{\frac{a_{2}+a_{1}}{a_{2}-a_{1}}} \tanh \left[ \pm \frac{1}{2} \sqrt{\frac{a_{2}^{2}-a_{1}^{2}}{a_{2}\left[k^{2}-\delta v-(1-\lambda) v^{2}\right]}}(x-v t)\right]\right\}
$$

with the constraints found in (6) and $a_{2}>\left|a_{1}\right|$. 


\subsection{Fourth Order Dispersion}

This section adds fourth order dispersion to the SG type equations. Equations with a fourth order dispersion term are commonly referred to as Boussinesq type equations by their resemblance to the Boussinesq equation:

$$
q_{t t}-q_{x x}+a\left(q^{2}\right)_{x x}+b q_{x x x x}=0
$$

The Boussinesq equation is an approximate equation for shallow water waves, similar to the well-known Korteveg de Vries (KdV) equation. Both equations model solitary waves, or solitons, along the surface of shallow water. For further discussion of the Boussinesq equation, see [14] [15].

\subsubsection{Sine-Gordon Equation}

The SGE with fourth-order dispersion is

$$
q_{t t}-k^{2} q_{x x}-d q_{x x x x}+a \sin q=0
$$

The Ansatz given by [16] is

$$
q(x, t)=8 \arctan \{A \exp [B(x-v t)]\}
$$

The 8 here means that this is in fact a double soliton. This can occur when two identical solitons interact and combine to form a single unit. A deeper discussion of this process can be found in [17]. The solution to the unperturbed dispersive SGE is

$$
q(x, t)=8 \arctan \left\{\exp \left[ \pm \sqrt{\frac{\frac{2}{3} a}{k^{2}-v^{2}}}\left(x-x_{0}-v t\right)\right]\right\}
$$

where

$$
v= \pm \sqrt{k^{2}-\sqrt{\frac{4}{3} a d}}
$$

with the following two restrictions on the parameters $a$ and $d$

$$
\begin{gathered}
a>0 \\
0<d<\frac{3 k^{4}}{4 a}
\end{gathered}
$$

This result agrees with the results found in [16]. The perturbed dispersive SGE is

$$
q_{t t}-k^{2} q_{x x}-d q_{x x x x}+a \sin q=R
$$

Using the same Ansatz from (11), the solution to the perturbed SGE with fourth order dispersion is

$$
q(x, t)=8 \arctan \left\{\exp \left[ \pm \sqrt{\frac{\frac{2}{3} a}{k^{2}-\delta v-(1-\lambda) v^{2}}}\left(x-x_{0}-v t\right)\right]\right\}
$$


where

$$
\begin{gathered}
\sigma=0 \\
\gamma=\beta v \\
v=\frac{-\delta \pm \sqrt{\delta^{2}+4(1-\lambda)\left(k^{2}-\sqrt{\frac{4 a}{3}(d+v)}\right)}}{2(1-\lambda)}
\end{gathered}
$$

which require the following inequalities to hold true

$$
\begin{gathered}
\lambda \neq 1 \\
a>0 \\
0<d+v<\frac{3}{4 a}\left(k^{2}+\frac{\delta^{2}}{4(1-\lambda)}\right)^{2}
\end{gathered}
$$

\subsubsection{SIne-Cosine-Gordon Equation}

The SCGE with fourth order dispersion is

$$
q_{t t}-k^{2} q_{x x}-d q_{x x x x}+a \sin q+b \cos q=0
$$

The Ansatz for this equation is

$$
q(x, t)=8 \arctan \{A \exp [B(x-v t)]\}+C
$$

The solution to the unperturbed dispersive SCGE is

$$
q(x, t)=8 \arctan \left\{\exp \left[ \pm \sqrt{\frac{\frac{2}{3} \sqrt{a^{2}+b^{2}}}{k^{2}-v^{2}}}\left(x-x_{0}-v t\right)\right]\right\}-\arctan \left(\frac{b}{a}\right)
$$

where

$$
v= \pm \sqrt{k^{2}-\sqrt{\frac{4}{3} d \sqrt{a^{2}+b^{2}}}}
$$

which puts the following restriction on the parameter $d$

$$
0<d<\frac{3 k^{4}}{4 \sqrt{a^{2}+b^{2}}}
$$

The perturbed dispersive SCGE is

$$
q_{t t}-k^{2} q_{x x}-d q_{x x x x}+a \sin q+b \cos q=R
$$

Using the previous Ansatz (12), the solution to the perturbed dispersive SCGE is

$$
q(x, t)=8 \arctan \left\{\exp \left[ \pm \sqrt{\frac{\frac{2}{3} \sqrt{a^{2}+b^{2}}}{k^{2}-\delta v-(1-\lambda) v^{2}}}\left(x-x_{0}-v t\right)\right]\right\}-\arctan \left(\frac{b}{a}\right)
$$

where 


$$
\begin{gathered}
\sigma=0 \\
\gamma=\beta v \\
v=\frac{-\delta \pm \sqrt{\delta^{2}+4(1-\lambda)\left(k^{2}-\sqrt{\frac{4}{3}(d+v) \sqrt{a^{2}+b^{2}}}\right)}}{2(1-\lambda)}
\end{gathered}
$$

which require the following inequalities

$$
0<d+v<\frac{3}{4 \sqrt{a^{2}+b^{2}}}\left(k^{2}+\frac{\delta^{2}}{4(1-\lambda)}\right)^{2}
$$

\subsubsection{Double Sine-Gordon Equation}

The DSGE with fourth order dispersion is

$$
q_{t t}-k^{2} q_{x x}-d q_{x x x x}+a_{1} \sin q+a_{2} \sin 2 q=0
$$

The Ansatz for this equation, due to [15], is

$$
q(x, t)=4 \arctan \{A \exp [B(x-v t)]\}
$$

The solution to the unperturbed dispersive DSGE is

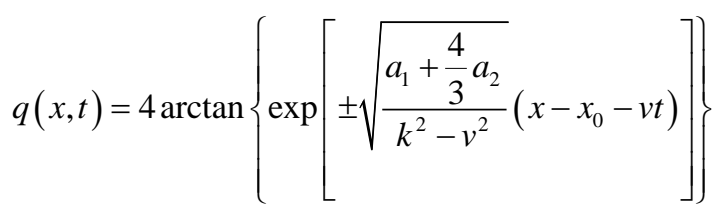

where

$$
v= \pm \sqrt{k^{2}-\left(a_{1}+\frac{4}{3} a_{2}\right) \sqrt{\frac{3 d}{2 a_{2}}}}
$$

which puts the following two restrictions on the parameters $a_{1}, a_{2}$, and $d$

$$
\begin{aligned}
a_{2} \cdot d & >0 \\
0<a_{1}+\frac{4}{3} a_{2} & <k^{2} \sqrt{\frac{2 a_{2}}{3 d}}
\end{aligned}
$$

The perturbed dispersive DSGE is

$$
q_{t t}-k^{2} q_{x x}-d q_{x x x x}+a_{1} \sin q+a_{2} \sin 2 q=R
$$

Using the Ansatz found in (13), the solution to the perturbed DSGE with fourth order dispersion is

$$
q(x, t)=8 \arctan \left\{\exp \left[ \pm \sqrt{\frac{a_{1}+\frac{4}{3} a_{2}}{k^{2}-\delta v-(1-\lambda) v^{2}}}\left(x-x_{0}-v t\right)\right]\right\}
$$

where

$$
\begin{gathered}
\sigma=0 \\
\gamma=\beta v
\end{gathered}
$$




$$
v=\frac{-\delta \pm\left\{\delta^{2}+4(1-\lambda)\left[k^{2}-\left(a_{1}+\frac{4}{3} a_{2}\right) \sqrt{\frac{3}{2 a_{2}}(d+v)}\right]\right\}^{\frac{1}{2}}}{2(1-\lambda)}
$$

which require the following inequalities to hold true

$$
\begin{gathered}
\lambda \neq 1 \\
a_{1}+\frac{4}{3} a_{2}>0 \\
0<\frac{d+v}{a_{2}}<\frac{2}{3}\left(\frac{k^{2}+\frac{\delta^{2}}{4(1-\lambda)}}{a_{1}+\frac{4}{3} a_{2}}\right)^{2}
\end{gathered}
$$

A portion of these results was already reported in [15]. They are presented here in a more complete manner than when they were first reported. It is also important to include these results here in this paper for the sake of completion.

\subsubsection{Double Sine-Cosine-Gordon Equation}

The double sine-cosine-Gordon equation (DSCGE) with fourth order dispersion is

$$
q_{t t}-k^{2} q_{x x}-d q_{x x x x}+a_{1} \sin q+b_{1} \cos q+a_{2} \sin 2 q+b_{2} \cos 2 q=0
$$

The Ansatz for this equation is

$$
q(x, t)=4 \arctan \{A \exp [B(x-v t)]\}+C
$$

It is first necessary to ensure

$$
\frac{a_{2}}{b_{2}}=\frac{a_{1}^{2}-b_{1}^{2}}{2 a_{1} b_{1}}
$$

Then the solution to the unperturbed dispersive DSCGE is

$$
\begin{aligned}
q(x, t)= & 4 \arctan \left\{\operatorname { e x p } \left[ \pm \sqrt{\frac{\left(a_{1}^{2}+b_{1}^{2}\right)^{\frac{3}{2}}+\frac{4}{3}\left[a_{2}\left(a_{1}^{2}-b_{1}^{2}\right)+2 a_{1} b_{1} b_{2}\right]}{\left(k^{2}-v^{2}\right)\left(a_{1}^{2}+b_{1}^{2}\right)}}\right.\right. \\
& \left.\left.\times\left(x-x_{0}-v t\right)\right]\right\}-\arctan \left(\frac{b_{1}}{a_{1}}\right)
\end{aligned}
$$

where

$$
v= \pm \sqrt{k^{2}-\left(a_{1}^{2}+b_{1}^{2}+\frac{4}{3} \cdot \frac{a_{2}\left(a_{1}^{2}-b_{1}^{2}\right)+2 a_{1} b_{1} b_{2}}{\sqrt{a_{1}^{2}+b_{1}^{2}}}\right) \sqrt{\frac{\frac{3}{2} d}{a_{2}\left(a_{1}^{2}-b_{1}^{2}\right)+2 a_{1} b_{1} b_{2}}}}
$$

which puts the following restrictions on the parameter $a_{1}, a_{2}, b_{1}, b_{2}$, and $d$ 


$$
\begin{gathered}
d\left[a_{2}\left(a_{1}^{2}-b_{1}^{2}\right)+2 a_{1} b_{1} b_{2}\right]>0 \\
0<\left(a_{1}^{2}+b_{1}^{2}\right)^{\frac{3}{2}}+\frac{4}{3}\left[a_{2}\left(a_{1}^{2}-b_{1}^{2}\right)+2 a_{1} b_{1} b_{2}\right] \\
\left(a_{1}^{2}+b_{1}^{2}\right)^{\frac{3}{2}}+\frac{4}{3}\left[a_{2}\left(a_{1}^{2}-b_{1}^{2}\right)+2 a_{1} b_{1} b_{2}\right]<k^{2} \sqrt{\frac{\left(a_{1}^{2}+b_{1}^{2}\right)\left[a_{2}\left(a_{1}^{2}-b_{1}^{2}\right)+2 a_{1} b_{1} b_{2}\right]}{\frac{3}{2} d}}
\end{gathered}
$$

The perturbed dispersive DSCGE is

$$
q_{t t}-k^{2} q_{x x}-d q_{x x x}+a_{1} \sin q+b_{1} \cos q+a_{2} \sin 2 q+b_{2} \cos 2 q=R
$$

Using the Ansatz found in (14) and the previously found relationships for $C$, $a_{1}, b_{1}, a_{2}$, and $b_{2}$, the solution to the perturbed dispersive DSCGE is

$$
\begin{aligned}
q(x, t)= & 4 \arctan \left\{\operatorname { e x p } \left[ \pm \sqrt{\frac{\left(a_{1}^{2}+b_{1}^{2}\right)^{\frac{3}{2}}+\frac{4}{3}\left[a_{2}\left(a_{1}^{2}-b_{1}^{2}\right)+2 a_{1} b_{1} b_{2}\right]}{\left[k^{2}-\delta v-(1-\lambda) v^{2}\right]\left(a_{1}^{2}+b_{1}^{2}\right)}}\right.\right. \\
& \left.\left.\times\left(x-x_{0}-v t\right)\right]\right\}-\arctan \left(\frac{b_{1}}{a_{1}}\right)
\end{aligned}
$$

where

$$
\begin{gathered}
\sigma=0 \\
\gamma=\beta \nu \\
v=\frac{1}{2(1-\lambda)}\left[-\delta \pm\left\{\delta^{2}+4(1-\lambda)\left[k^{2}-\left(a_{1}^{2}+b_{1}^{2}\right.\right.\right.\right. \\
\left.\left.+\frac{4}{3} \cdot \frac{a_{2}\left(a_{1}^{2}-b_{1}^{2}\right)+2 a_{1} b_{1} b_{2}}{\sqrt{a_{1}^{2}+b_{1}^{2}}}\right) \sqrt{\left.\frac{\frac{3}{2}(d+v)}{a_{2}\left(a_{1}^{2}-b_{1}^{2}\right)+2 a_{1} b_{1} b_{2}}\right]}\right)
\end{gathered}
$$

in which case the following inequalities must also hold true

$$
\begin{gathered}
\lambda \neq 1 \\
{\left[a_{2}\left(a_{1}^{2}-b_{1}^{2}\right)+2 a_{1} b_{1} b_{2}\right](d+v)>0} \\
0<\left(a_{1}^{2}+b_{1}^{2}\right)^{\frac{3}{2}}+\frac{4}{3}\left[a_{2}\left(a_{1}^{2}-b_{1}^{2}\right)+2 a_{1} b_{1} b_{2}\right] \\
a_{1}^{2}+b_{1}^{2}+\frac{4}{3} \cdot \frac{a_{2}\left(a_{1}^{2}-b_{1}^{2}\right)+2 a_{1} b_{1} b_{2}}{\sqrt{a_{1}^{2}+b_{1}^{2}}}<\left(k^{2}+\frac{\delta^{2}}{4(1-\lambda)}\right) \sqrt{\frac{a_{2}\left(a_{1}^{2}-b_{1}^{2}\right)+2 a_{1} b_{1} b_{2}}{\frac{3}{2}(d+v)}}
\end{gathered}
$$




\subsection{Sixth Order Dispersion}

Higher order dispersion terms mainly come about from stronger interactions of the highly discretized SGE, see [19]. Just as the fourth order dispersion term gave way to double solitons, the sixth order dispersion term will yield triple solitons. For further discussion of the discretized SGE and these triple solitons, see [17].

\subsubsection{Sine-Gordon Equation}

The SGE with sixth order dispersion is

$$
q_{t t}-k^{2} q_{x x}-d_{1} q_{x x x x}-d_{2} q_{x x x x x}+a \sin q=0
$$

The Ansatz for this equation, found in [16], is

$$
q(x, t)=12 \arctan \{A \exp [B(x-v t)]\}
$$

The 12 here means this is a triple soliton. The solution to the unperturbed highly dispersive SGE is

$$
q(x, t)=12 \arctan \left\{\exp \left[ \pm \sqrt{\frac{\frac{23}{45} a}{k^{2}-v^{2}}}\left(x-x_{0}-v t\right)\right]\right\}
$$

where

$$
v= \pm \sqrt{k^{2}-\frac{23}{30} \sqrt{a d_{1}}}
$$

which puts the following restrictions on the parameters $a, d_{1}$, and $d_{2}$

$$
\begin{gathered}
a>0 \\
d_{2}=\frac{3}{20} \sqrt{\frac{d_{1}^{3}}{a}} \\
0<d_{1}<\frac{900 k^{4}}{529 a}
\end{gathered}
$$

The solution to the unperturbed highly dispersive SGE is

The perturbed SGE with sixth order dispersion is

$$
q_{t t}-k^{2} q_{x x}-d_{1} q_{x x x x}-d_{2} q_{x x x x x}+a \sin q=R
$$

Using the same Ansatz as in (15) yields

$$
\begin{aligned}
0= & B(\beta v-\gamma)\left(E+5 E^{3}+10 E^{5}+10 E^{7}+5 E^{9}+E^{11}\right) \\
& -B^{2}\left(k^{2}-\delta v-(1-\lambda) v^{2}\right)\left(E+3 E^{3}+2 E^{5}-2 E^{7}-3 E^{9}-E^{11}\right) \\
& +B^{3} \sigma v\left(E-3 E^{3}-14 E^{5}-14 E^{7}-3 E^{9}+E^{11}\right) \\
& -B^{4}\left(d_{1}+v\right)\left(E-21 E^{3}-22 E^{5}+22 E^{7}+21 E^{9}-E^{11}\right) \\
& -d_{2} B^{6}\left(E-237 E^{3}+1682 E^{5}-1682 E^{7}+237 E^{9}-E^{11}\right) \\
& +\frac{a}{3}\left(3 E-55 E^{3}+198 E^{5}-198 E^{7}+55 E^{9}-3 E^{11}\right)
\end{aligned}
$$


This is equivalent to the matrix equation

$$
\left(\begin{array}{rrrrrr}
1 & 1 & 1 & 1 & 1 & 3 \\
5 & 3 & -3 & -21 & -237 & -55 \\
10 & 2 & -14 & -22 & 1682 & 198 \\
10 & -2 & -14 & 22 & -1682 & -198 \\
5 & -3 & -3 & 21 & 237 & 55 \\
1 & -1 & 1 & -1 & -1 & -3
\end{array}\right)\left(\begin{array}{l}
c_{1} \\
c_{2} \\
c_{3} \\
c_{4} \\
c_{5} \\
c_{6}
\end{array}\right)=\left(\begin{array}{l}
0 \\
0 \\
0 \\
0 \\
0 \\
0
\end{array}\right)
$$

where $c_{1}=B(\beta v-\gamma), \quad c_{2}=-B^{2}\left[k^{2}-\delta v-(1-\lambda) v^{2}\right], \quad c_{3}=B^{3} \sigma v$, $c_{4}=-B^{4}\left(d_{1}+v\right), \quad c_{5}=-d_{2} B^{6}$, and $c_{6}=a / 3$. Solving the above system gives us

$$
\begin{gathered}
\sigma=0 \\
\gamma=\beta v \\
d_{2}=\frac{3}{20} \sqrt{\frac{\left(d_{1}+v\right)^{3}}{a}} \\
B= \pm \sqrt{\frac{\frac{23}{45} a}{k^{2}-\delta v-(1-\lambda) v^{2}}} \\
v=\frac{-\delta \pm \sqrt{\delta^{2}+4(1-\lambda)\left(k^{2}-\frac{23}{30} \sqrt{a\left(d_{1}+v\right)}\right)}}{2(1-\lambda)}
\end{gathered}
$$

with constraints

$$
\begin{gathered}
\lambda \neq 1 \\
a>0 \\
0<d_{1}+v<\frac{900}{529 a}\left(k^{2}+\frac{\delta^{2}}{4(1-\lambda)}\right)^{2}
\end{gathered}
$$

The solution to the perturbed highly dispersive SGE is

$$
q(x, t)=12 \arctan \left\{\exp \left[ \pm \sqrt{\frac{\frac{23}{45} a}{k^{2}-\delta v-(1-\lambda) v^{2}}}\left(x-x_{0}-v t\right)\right]\right\}
$$

\subsubsection{Sine-Cosine-Gordon Equation}

The SCGE with sixth order dispersion is

$$
q_{t t}-k^{2} q_{x x}-d_{1} q_{x x x x}-d_{2} q_{x x x x x x}+a \sin q+b \cos q=0
$$

The Ansatz for this equation is

$$
q(x, t)=12 \arctan \{A \exp [B(x-v t)]\}+C
$$

First, set $C=-\arctan (b / a)$. Then (16) and (17) give us 


$$
\begin{aligned}
0= & -\left(k^{2}-v^{2}\right) B^{2}\left(E+3 E^{3}+2 E^{5}-2 E^{7}-3 E^{9}-E^{11}\right) \\
& -d_{1} B^{4}\left(E-21 E^{3}-22 E^{5}+22 E^{7}+21 E^{9}-E^{11}\right) \\
& -d_{2} B^{6}\left(E-237 E^{3}+1682 E^{5}-1682 E^{7}+237 E^{9}-E^{11}\right) \\
& +\frac{1}{3} \sqrt{a^{2}+b^{2}}\left(3 E-55 E^{3}+198 E^{5}-198 E^{7}+55 E^{9}-3 E^{11}\right)
\end{aligned}
$$

Solving the same system of equations from Section 3.3.1 leads to the similar pair of relations

$$
\begin{gathered}
B=\sqrt{\frac{\frac{23}{45} \sqrt{a^{2}+b^{2}}}{k^{2}-v^{2}}} \\
v=\sqrt{k^{2}-\frac{23}{30}\left(a^{2}+b^{2}\right)^{\frac{1}{4}} \sqrt{d_{1}}}
\end{gathered}
$$

which put the following pair of restrictions on parameters $d_{1}$ and $d_{2}$

$$
\begin{gathered}
d_{2}=\frac{3}{20} \cdot \frac{d_{1}^{\frac{3}{2}}}{\left(a^{2}+b^{2}\right)^{\frac{1}{4}}} \\
0<d_{1}<\frac{900 k^{4}}{529 \sqrt{a^{2}+b^{2}}}
\end{gathered}
$$

The solution to the unperturbed highly dispersive SCGE is

$$
q(x, t)=12 \arctan \left\{A \exp \left[\sqrt{\frac{\frac{23}{45} \sqrt{a^{2}+b^{2}}}{k^{2}-v^{2}}}\left(x-x_{0}-v t\right)\right]\right\}-\arctan \left(\frac{b}{a}\right)
$$

The perturbed SCGE with sixth order dispersion is

$$
q_{t t}-k^{2} q_{x x}-d_{1} q_{x x x x}-d_{2} q_{x x x x x}+a \sin q+b \cos q=R
$$

Using the same Ansatz from (17), we have

$$
\begin{aligned}
0= & B(\beta v-\gamma)\left(E+5 E^{3}+10 E^{5}+10 E^{7}+5 E^{9}+E^{11}\right) \\
& -B^{2}\left(k^{2}-\delta v-(1-\lambda) v^{2}\right)\left(E+3 E^{3}+2 E^{5}-2 E^{7}-3 E^{9}-E^{11}\right) \\
& +B^{3} \sigma v\left(E-3 E^{3}-14 E^{5}-14 E^{7}-3 E^{9}+E^{11}\right) \\
& -B^{4}\left(d_{1}+v\right)\left(E-21 E^{3}-22 E^{5}+22 E^{7}+21 E^{9}-E^{11}\right) \\
& -d_{2} B^{6}\left(E-237 E^{3}+1682 E^{5}-1682 E^{7}+237 E^{9}-E^{11}\right) \\
& +\frac{1}{3} \sqrt{a^{2}+b^{2}}\left(3 E-55 E^{3}+198 E^{5}-198 E^{7}+55 E^{9}-3 E^{11}\right)
\end{aligned}
$$

This leads to the same matrix equation from Section 3.3.1 where $c_{1}=B(\beta v-\gamma), \quad c_{2}=-B^{2}\left[k^{2}-\delta v-(1-\lambda) v^{2}\right], \quad c_{3}=B^{3} \sigma v, \quad c_{4}=-B^{4}\left(d_{1}+v\right)$, $c_{5}=-d_{2} B^{6}$, and $c_{6}=\sqrt{a^{2}+b^{2}} / 3$. The relationships now are

$$
\begin{gathered}
\sigma=0 \\
\gamma=\beta v
\end{gathered}
$$




$$
\begin{gathered}
d_{2}=\frac{3}{20} \frac{\left(d_{1}+v\right)^{\frac{3}{2}}}{\left(a^{2}+b^{2}\right)^{\frac{1}{4}}} \\
B= \pm \sqrt{\frac{\frac{23}{45} \sqrt{a^{2}+b^{2}}}{k^{2}-\delta v-(1-\lambda) v^{2}}} \\
v=\frac{-\delta \pm \sqrt{\delta^{2}+4(1-\lambda)\left(k^{2}-\frac{23}{30}\left(a^{2}+b^{2}\right)^{\frac{1}{4}} \sqrt{d_{1}+v}\right)}}{2(1-\lambda)}
\end{gathered}
$$

with constraints

$$
\lambda \neq 1
$$

$$
0<d_{1}+v<\frac{900}{529 \sqrt{a^{2}+b^{2}}}\left(k^{2}+\frac{\delta^{2}}{4(1-\lambda)}\right)^{2}
$$

The solution to the perturbed highly dispersive SCGE is

$$
q(x, t)=12 \arctan \left\{\exp \left[ \pm \sqrt{\frac{\frac{23}{45} \sqrt{a^{2}+b^{2}}}{k^{2}-\delta v-(1-\lambda) v^{2}}}\left(x-x_{0}-v t\right)\right]\right\}-\arctan \left(\frac{b}{a}\right)
$$

\subsubsection{Triple Sine-Gordon Equation}

The triple sine-Gordon equation (TSGE) with sixth order dispersion is

$$
q_{t t}-k^{2} q_{x x}-d_{1} q_{x x x x}-d_{2} q_{x x x x x x}+a_{1} \sin q+a_{2} \sin 2 q+a_{3} \sin 3 q=0
$$

The Ansatz for (18), due to [16], is

$$
q(x, t)=4 \arctan \{A \exp [B(x-v t)]\}
$$

Plugging (19) into (18) gives us

$$
\begin{aligned}
0= & {\left[a_{1}-B^{2}\left(k^{2}-v^{2}\right)\right]\left(E+3 E^{3}+2 E^{5}-2 E^{7}-3 E^{9}-E^{11}\right) } \\
& -d_{1} B^{4}\left(E-21 E^{3}-22 E^{5}+22 E^{7}+21 E^{9}-E^{11}\right) \\
& -d_{2} B^{6}\left(E-237 E^{3}+1682 E^{5}-1682 E^{7}+237 E^{9}-E^{11}\right) \\
& +2 a_{2}\left(E-5 E^{3}-6 E^{5}+6 E^{7}+5 E^{9}-E^{11}\right) \\
& +a_{3}\left(3 E-55 E^{3}+198 E^{5}-198 E^{7}+55 E^{9}-3 E^{11}\right)
\end{aligned}
$$

This leads to the system of equations according to the powers of $E$

$$
\left(\begin{array}{rrrrr}
1 & 1 & 1 & 1 & 3 \\
3 & -21 & -237 & -5 & -55 \\
2 & -22 & 1682 & -6 & 198 \\
-2 & 22 & -1682 & 6 & -198 \\
-3 & 21 & 237 & 5 & 55 \\
-1 & -1 & -1 & -1 & -3
\end{array}\right)\left(\begin{array}{l}
c_{1} \\
c_{2} \\
c_{3} \\
c_{4} \\
c_{5}
\end{array}\right)=\left(\begin{array}{l}
0 \\
0 \\
0 \\
0 \\
0 \\
0
\end{array}\right)
$$

where $c_{1}=a-B^{2}\left(k^{2}-v^{2}\right), \quad c_{2}=-d_{1} B^{4}, \quad c_{3}=-d_{2} B^{6}, \quad c_{4}=2 a_{2}$, and $c_{5}=a_{3}$. 
Solving the above system leads to the following relations

$$
\begin{gathered}
B= \pm \sqrt{\frac{a_{1}+\frac{4}{3} a_{2}+\frac{23}{15} a_{3}}{k^{2}-v^{2}}} \\
v= \pm \sqrt{k^{2}-\left(a_{1}+\frac{4}{3} a_{2}+\frac{23}{15} a_{3}\right) \sqrt{\frac{\frac{3}{2} d_{1}}{a_{2}+2 a_{3}}}} \\
d_{2}=\frac{2 a_{3}}{15}\left(\frac{\frac{3}{2} d_{1}}{a_{2}+2 a_{3}}\right)^{\frac{3}{2}}
\end{gathered}
$$

which put the following four restrictions on the parameters $a_{1}, a_{2}, a_{3}, d_{1}$, and $d_{2}$

$$
\begin{gathered}
d_{1}\left(a_{2}+2 a_{3}\right)>0 \\
0<a_{1}+\frac{4}{3} a_{2}+\frac{23}{15} a_{3}<k^{2} \sqrt{\frac{\frac{3}{2} d_{1}}{a_{2}+2 a_{3}}}
\end{gathered}
$$

The solution to the unperturbed highly dispersive TSGE is

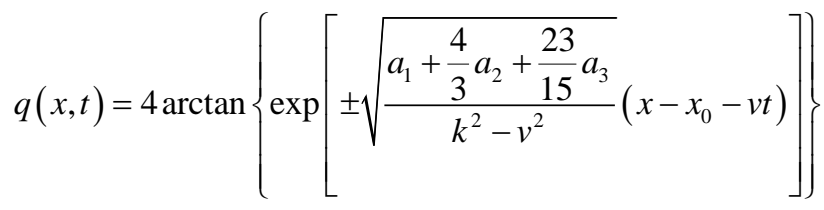

The perturbed TSGE with sixth order dispersion is

$$
q_{t t}-k^{2} q_{x x}-d_{1} q_{x x x x}-d_{2} q_{x x x x x}+a_{1} \sin q+a_{2} \sin 2 q+a_{3} \sin 3 q=R
$$

Using the Ansatz found in (19) gives

$$
\begin{aligned}
0= & B(\beta v-\gamma)\left(E+5 E^{3}+10 E^{5}+10 E^{7}+5 E^{9}+E^{11}\right) \\
& +\left[a_{1}-B^{2}\left(k^{2}-\delta v-(1-\lambda) v^{2}\right)\right]\left(E+3 E^{3}+2 E^{5}-2 E^{7}-3 E^{9}-E^{11}\right) \\
& +B^{3} \sigma v\left(E-3 E^{3}-14 E^{5}-14 E^{7}-3 E^{9}+E^{11}\right) \\
& -B^{4}\left(d_{1}+v\right)\left(E-21 E^{3}-22 E^{5}+22 E^{7}+21 E^{9}-E^{11}\right) \\
& -d_{2} B^{6}\left(E-237 E^{3}+1682 E^{5}-1682 E^{7}+237 E^{9}-E^{11}\right) \\
& +2 a_{2}\left(E-5 E^{3}-6 E^{5}+6 E^{7}+5 E^{9}-E^{11}\right) \\
& +a_{3}\left(3 E-55 E^{3}+198 E^{5}-198 E^{7}+55 E^{9}-3 E^{11}\right)
\end{aligned}
$$

This is equivalent to the matrix equation

$$
\left(\begin{array}{rrrrrrr}
1 & 1 & 1 & 1 & 1 & 1 & 3 \\
5 & 3 & -3 & -21 & -237 & -5 & -55 \\
10 & 2 & -14 & -22 & 1682 & -6 & 198 \\
10 & -2 & -14 & 22 & -1682 & 6 & -198 \\
5 & -3 & -3 & 21 & 237 & 5 & 55 \\
1 & -1 & 1 & -1 & -1 & -1 & -3
\end{array}\right)\left(\begin{array}{l}
c_{1} \\
c_{2} \\
c_{3} \\
c_{4} \\
c_{5} \\
c_{6} \\
c_{7}
\end{array}\right)=\left(\begin{array}{l}
0 \\
0 \\
0 \\
0 \\
0 \\
0
\end{array}\right)
$$


where $c_{1}=B(\beta v-\gamma), \quad c_{2}=a_{1}-B^{2}\left[k^{2}-\delta v-(1-\lambda) v^{2}\right], \quad c_{3}=B^{3} \sigma v$, $c_{4}=-B^{4}\left(d_{1}+v\right), \quad c_{5}=-d_{2} B^{6}, \quad c_{6}=2 a_{2}$, and $c_{7}=a_{3}$. Solving this system results in the following equalities

$$
\begin{gathered}
\sigma=0 \\
\gamma=\beta v \\
d_{2}=\frac{2 a_{3}\left(\frac{3}{2} \cdot \frac{d_{1}+v}{a_{2}+2 a_{3}}\right)^{\frac{3}{2}}}{B= \pm \sqrt{\frac{a_{1}+\frac{4}{3} a_{2}+\frac{23}{15} a_{3}}{k^{2}-\delta v-(1-\lambda) v^{2}}}} \\
v=\frac{-\delta \pm \sqrt{\delta^{2}+4(1-\lambda)\left[k^{2}-\left(a_{1}+\frac{4}{3} a_{2}+\frac{23}{15} a_{3}\right) \sqrt{\frac{3}{2} \cdot \frac{d_{1}+v}{a_{2}+2 a_{3}}}\right]}}{2(1-\lambda)}
\end{gathered}
$$

with constraints

$$
\begin{gathered}
\lambda \neq 1 \\
d_{1}\left(a_{2}+2 a_{3}\right)>0 \\
0<a_{1}+\frac{4}{3} a_{2}+\frac{23}{15} a_{3}<\sqrt{\frac{2}{3} \cdot \frac{a_{2}+2 a_{3}}{d_{1}+v}}\left(k^{2}+\frac{\delta^{2}}{4(1-\lambda)}\right)
\end{gathered}
$$

The solution to the perturbed highly dispersive TSGE is

$$
q(x, t)=4 \arctan \left\{\exp \left[ \pm \sqrt{\frac{a_{1}+\frac{4}{3} a_{2}+\frac{23}{15} a_{3}}{k^{2}-\delta v-(1-\lambda) v^{2}}}\left(x-x_{0}-v t\right)\right]\right\}
$$

\subsubsection{Triple Sine-Cosine-Gordon Equation}

The triple sine-cosine-Gordon (TSCGE) equation with sixth order dispersion is

$$
\begin{aligned}
& q_{t t}-k^{2} q_{x x}-d_{1} q_{x x x x}-d_{2} q_{x x x x x}+a_{1} \sin q+b_{1} \cos q \\
& +a_{2} \sin 2 q+b_{2} \cos 2 q+a_{3} \sin 3 q+b_{3} \cos 3 q=0
\end{aligned}
$$

The Ansatz for (20) is

$$
q(x, t)=4 \arctan \{A \exp [B(x-v t)]\}+C
$$

It is necessary to set $C=-\arctan \left(b_{1} / a_{1}\right)$ and to ensure both

$$
\frac{a_{2}}{b_{2}}=\frac{a_{1}^{2}-b_{1}^{2}}{2 a_{1} b_{1}}
$$

and

$$
\frac{a_{3}}{b_{3}}=-\frac{a_{1}}{b_{1}} \cdot \frac{a_{1}^{3}-3 b_{1}^{3}}{b_{1}^{3}-3 a_{1}^{3}}
$$

to eliminate the even exponents of $E$ from the cosine terms. These make the fol- 
lowing three simplifications

$$
\begin{gathered}
a_{1} \sin q+b_{1} \cos q=\sqrt{a_{1}^{2}+b_{1}^{2}} \sin q \\
a_{2} \sin 2 q+b_{2} \cos 2 q=\frac{a_{2}\left(a_{1}^{2}-b_{1}^{2}\right)+2 a_{1} b_{1} b_{2}}{a_{1}^{2}+b_{1}^{2}} \sin 2 q \\
a_{3} \sin 3 q+b_{3} \cos 3 q=\frac{a_{1} a_{3}\left(a_{1}^{2}-3 b_{1}^{2}\right)-b_{1} b_{3}\left(b_{1}^{2}-3 a_{1}^{2}\right)}{\left(a_{1}^{2}+b_{1}^{2}\right)^{\frac{3}{2}}} \sin 3 q
\end{gathered}
$$

Combining (20) into (21) yields

$$
\begin{aligned}
0= & {\left[\sqrt{a_{1}^{2}+b_{1}^{2}}-B^{2}\left(k^{2}-v^{2}\right)\right]\left(E+3 E^{3}+2 E^{5}-2 E^{7}-3 E^{9}-E^{11}\right) } \\
& -d_{1} B^{4}\left(E-21 E^{3}-22 E^{5}+22 E^{7}+21 E^{9}-E^{11}\right) \\
& -d_{2} B^{6}\left(E-237 E^{3}+1682 E^{5}-1682 E^{7}+237 E^{9}-E^{11}\right) \\
& +2 \frac{a_{2}\left(a_{1}^{2}-b_{1}^{2}\right)+2 a_{1} b_{1} b_{2}}{a_{1}^{2}+b_{1}^{2}}\left(E-5 E^{3}-6 E^{5}+6 E^{7}+5 E^{9}-E^{11}\right) \\
& +\frac{a_{1} a_{3}\left(a_{1}^{2}-3 b_{1}^{2}\right)-b_{1} b_{3}\left(b_{1}^{2}-3 a_{1}^{2}\right)}{\left(a_{1}^{2}+b_{1}^{2}\right)^{\frac{3}{2}}}\left(3 E-55 E^{3}+198 E^{5}\right. \\
& \left.-198 E^{7}+55 E^{9}-3 E^{11}\right)
\end{aligned}
$$

This gives rise to the same matrix equation from 3.3.3, where this time $c_{1}=\sqrt{a_{1}^{2}+b_{1}^{2}}-B^{2}\left(k^{2}-v^{2}\right), \quad c_{2}=-d_{1} B^{4}, \quad c_{3}=-d_{2} B^{6}$, $c_{4}=2\left[a_{2}\left(a_{1}^{2}-b_{1}^{2}\right)+2 a_{1} b_{1} b_{2}\right] /\left(a_{1}^{2}+b_{1}^{2}\right)$, and $c_{5}=\left[a_{1} a_{3}\left(a_{1}^{2}-3 b_{1}^{2}\right)-b_{1} b_{3}\left(b_{1}^{2}-3 a_{1}^{2}\right)\right] /\left(a_{1}^{2}+b_{1}^{2}\right)^{\frac{3}{2}}$. The solution to that system gives us

$$
\begin{aligned}
B= & \pm \frac{1}{\sqrt{k^{2}-v^{2}}\left(a_{1}^{2}+b_{1}^{2}\right)^{\frac{3}{4}}}\left\{\left(a_{1}^{2}+b_{1}^{2}\right)^{2}+\frac{4}{3}\left[a_{2}\left(a_{1}^{2}-b_{1}^{2}\right)+2 a_{1} b_{1} b_{2}\right] \sqrt{a_{1}^{2}+b_{1}^{2}}\right. \\
+ & \left.\frac{23}{15}\left[a_{1} a_{3}\left(a_{1}^{2}-3 b_{1}^{2}\right)-b_{1} b_{3}\left(b_{1}^{2}-3 a_{1}^{2}\right)\right]\right\}^{\frac{1}{2}} \\
v= & \pm\left\{k^{2}-\left(a_{1}^{2}+b_{1}^{2}+\frac{4}{3} \frac{a_{2}\left(a_{1}^{2}-b_{1}^{2}\right)+2 a_{1} b_{1} b_{2}}{\sqrt{a_{1}^{2}+b_{1}^{2}}}\right.\right. \\
& \left.+\frac{23}{15} \frac{a_{1} a_{3}\left(a_{1}^{2}-3 b_{1}^{2}\right)-b_{1} b_{3}\left(b_{1}^{2}-3 a_{1}^{2}\right)}{a_{1}^{2}+b_{1}^{2}}\right) \\
& \left.\times \sqrt{\frac{\frac{3}{2} d_{1}}{a_{2}\left(a_{1}^{2}-b_{1}^{2}\right)+2 a_{1} b_{1} b_{2}+2 \frac{a_{1} a_{3}\left(a_{1}^{2}-3 b_{1}^{2}\right)-b_{1} b_{3}\left(b_{1}^{2}-3 a_{1}^{2}\right)}{\sqrt{a_{1}^{2}+b_{1}^{2}}}}}\right\}^{\frac{1}{2}}
\end{aligned}
$$




$$
\begin{aligned}
d_{2} & =\frac{2}{15}\left[a_{1} a_{3}\left(a_{1}^{2}-3 b_{1}^{2}\right)-b_{1} b_{3}\left(b_{1}^{2}-3 a_{1}^{2}\right)\right] \\
& \times\left(\frac{\frac{3}{2} d_{1}}{a_{2}\left(a_{1}^{2}-b_{1}^{2}\right)+2 a_{1} b_{1} b_{2}+2 \frac{a_{1} a_{3}\left(a_{1}^{2}-3 b_{1}^{2}\right)-b_{1} b_{3}\left(b_{1}^{2}-3 a_{1}^{2}\right)}{\sqrt{a_{1}^{2}+b_{1}^{2}}}}\right)^{\frac{3}{2}}
\end{aligned}
$$

and this gives way to the following constraints on the parameters $a_{1}, a_{2}, a_{3}$, $b_{1}, b_{2}, b_{3}, d_{1}$, and $d_{2}$

$$
\begin{aligned}
& d_{1}\left[a_{2}\left(a_{1}^{2}-b_{1}^{2}\right)+2 a_{1} b_{1} b_{2}+2 \frac{a_{1} a_{3}\left(a_{1}^{2}-3 b_{1}^{2}\right)-b_{1} b_{3}\left(b_{1}^{2}-3 a_{1}^{2}\right)}{\sqrt{a_{1}^{2}+b_{1}^{2}}}\right]>0 \\
0 & <a_{1}^{2}+b_{1}^{2}+\frac{4}{3} \frac{a_{2}\left(a_{1}^{2}-b_{1}^{2}\right)+2 a_{1} b_{1} b_{2}}{\sqrt{a_{1}^{2}+b_{1}^{2}}}+\frac{23}{15} \frac{a_{1} a_{3}\left(a_{1}^{2}-3 b_{1}^{2}\right)-b_{1} b_{3}\left(b_{1}^{2}-3 a_{1}^{2}\right)}{a_{1}^{2}+b_{1}^{2}} \\
< & \sqrt{\frac{a_{2}\left(a_{1}^{2}-b_{1}^{2}\right)+2 a_{1} b_{1} b_{2}+2 \frac{a_{1} a_{3}\left(a_{1}^{2}-3 b_{1}^{2}\right)-b_{1} b_{3}\left(b_{1}^{2}-3 a_{1}^{2}\right)}{\sqrt{a_{1}^{2}+b_{1}^{2}}}}{\frac{3}{2} d_{1}}}
\end{aligned}
$$

The solution to the unperturbed highly dispersive TSCGE is

$$
\begin{aligned}
q(x, t)= & 4 \arctan \left\{\operatorname { e x p } \left[ \pm\left\{\left(a_{1}^{2}+b_{1}^{2}\right)^{2}+\frac{4}{3}\left[a_{2}\left(a_{1}^{2}-b_{1}^{2}\right)+2 a_{1} b_{1} b_{2}\right] \sqrt{a_{1}^{2}+b_{1}^{2}}\right.\right.\right. \\
& \left.\left.\left.+\frac{23}{15}\left[a_{1} a_{3}\left(a_{1}^{2}-3 b_{1}^{2}\right)-b_{1} b_{3}\left(b_{1}^{2}-3 a_{1}^{2}\right)\right]\right\} \frac{\left(x-x_{0}-v t\right)}{\left(a_{1}^{2}+b_{1}^{2}\right)^{\frac{3}{4}} \sqrt{k^{2}-v^{2}}}\right]\right\}
\end{aligned}
$$

The perturbed TSCGE with sixth order dispersion is

$$
\begin{aligned}
& q_{t t}-k^{2} q_{x x}-d_{1} q_{x x x x}-d_{2} q_{x x x x x x}+a_{1} \sin q+b_{1} \cos q \\
& +a_{2} \sin 2 q+b_{2} \cos 2 q+a_{3} \sin 3 q+b_{3} \cos 3 q=0
\end{aligned}
$$

Using the previous Ansatz from (21) gives the governing equation

$$
\begin{aligned}
0= & B(\beta v-\gamma)\left(E+5 E^{3}+10 E^{5}+10 E^{7}+5 E^{9}+E^{11}\right) \\
& +\left[\sqrt{a_{1}^{2}+b_{1}^{2}}-B^{2}\left(k^{2}-\delta v-(1-\lambda) v^{2}\right)\right]\left(E+3 E^{3}+2 E^{5}-2 E^{7}\right. \\
& \left.-3 E^{9}-E^{11}\right)+B^{3} \sigma v\left(E-3 E^{3}-14 E^{5}-14 E^{7}-3 E^{9}+E^{11}\right) \\
& -B^{4}\left(d_{1}+v\right)\left(E-21 E^{3}-22 E^{5}+22 E^{7}+21 E^{9}-E^{11}\right) \\
& -d_{2} B^{6}\left(E-237 E^{3}+1682 E^{5}-1682 E^{7}+237 E^{9}-E^{11}\right) \\
& +2 \frac{a_{2}\left(a_{1}^{2}-b_{1}^{2}\right)+2 a_{1} b_{1} b_{2}}{a_{1}^{2}+b_{1}^{2}}\left(E-5 E^{3}-6 E^{5}+6 E^{7}+5 E^{9}-E^{11}\right) \\
& +\frac{a_{1} a_{3}\left(a_{1}^{2}-3 b_{1}^{2}\right)-b_{1} b_{3}\left(b_{1}^{2}-3 a_{1}^{2}\right)}{\left(a_{1}^{2}+b_{1}^{2}\right)^{\frac{3}{2}}}\left(3 E-55 E^{3}+198 E^{5}\right. \\
& \left.-198 E^{7}+55 E^{9}-3 E^{11}\right)
\end{aligned}
$$


This is again equivalent to the matrix equation from 3.3.3, where this time $c_{1}=B(\beta v-\gamma), \quad c_{2}=\sqrt{a_{1}^{2}+b_{1}^{2}}-B^{2}\left[k^{2}-\delta v-(1-\lambda) v^{2}\right], \quad c_{3}=B^{3} \sigma v$, $c_{4}=-B^{4}\left(d_{1}+v\right), \quad c_{5}=-d_{2} B^{6}, \quad c_{6}=2\left[a_{2}\left(a_{1}^{2}-b_{1}^{2}\right)+2 a_{1} b_{1} b_{2}\right] /\left(a_{1}^{2}+b_{1}^{2}\right)$, and $c_{7}=\left[a_{1} a_{3}\left(a_{1}^{2}-3 b_{1}^{2}\right)-b_{1} b_{3}\left(b_{1}^{2}-3 a_{1}^{2}\right)\right] /\left(a_{1}^{2}+b_{1}^{2}\right)^{\frac{3}{2}}$. Solving that system results in the following equalities

$$
\begin{aligned}
& \sigma=0 \\
& \gamma=\beta v \\
& d_{2}=\frac{2}{15}\left[a_{1} a_{3}\left(a_{1}^{2}-3 b_{1}^{2}\right)-b_{1} b_{3}\left(b_{1}^{2}-3 a_{1}^{2}\right)\right] \\
& \times\left(\frac{\frac{3}{2}\left(d_{1}+v\right)}{a_{2}\left(a_{1}^{2}-b_{1}^{2}\right)+2 a_{1} b_{1} b_{2}+2 \frac{a_{1} a_{3}\left(a_{1}^{2}-3 b_{1}^{2}\right)-b_{1} b_{3}\left(b_{1}^{2}-3 a_{1}^{2}\right)}{\sqrt{a_{1}^{2}+b_{1}^{2}}}}\right)^{\frac{3}{2}} \\
& B=\frac{ \pm 1}{\sqrt{k^{2}-\delta v-(1-\lambda) v^{2}}\left(a_{1}^{2}+b_{1}^{2}\right)^{\frac{3}{4}}}\left\{\left(a_{1}^{2}+b_{1}^{2}\right)^{2}+\frac{4}{3}\left[a_{2}\left(a_{1}^{2}-b_{1}^{2}\right)+2 a_{1} b_{1} b_{2}\right]\right. \\
& \left.\times \sqrt{a_{1}^{2}+b_{1}^{2}}+\frac{23}{15}\left[a_{1} a_{3}\left(a_{1}^{2}-3 b_{1}^{2}\right)-b_{1} b_{3}\left(b_{1}^{2}-3 a_{1}^{2}\right)\right]\right\}^{\frac{1}{2}} \\
& v=\frac{1}{2(1-\lambda)}\left(-\delta \pm\left\{\delta^{2}+4(1-\lambda)\left[k^{2}-\left(a_{1}^{2}+b_{1}^{2}\right.\right.\right.\right. \\
& \left.+\frac{4}{3} \frac{a_{2}\left(a_{1}^{2}-b_{1}^{2}\right)+2 a_{1} b_{1} b_{2}}{\sqrt{a_{1}^{2}+b_{1}^{2}}}+\frac{23}{15} \frac{a_{1} a_{3}\left(a_{1}^{2}-3 b_{1}^{2}\right)-b_{1} b_{3}\left(b_{1}^{2}-3 a_{1}^{2}\right)}{a_{1}^{2}+b_{1}^{2}}\right)
\end{aligned}
$$

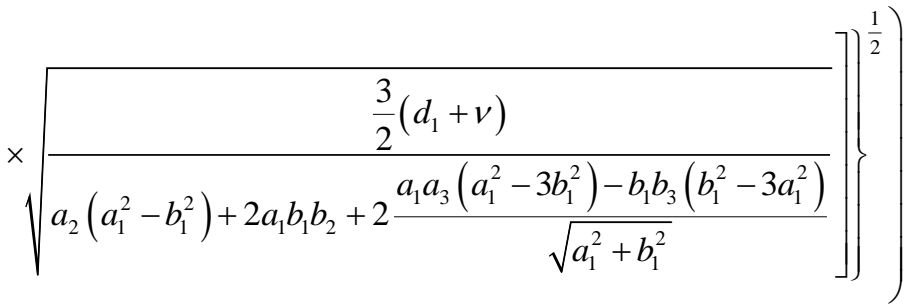

with constraints

$$
\begin{gathered}
\lambda \neq 1 \\
d_{1}\left[a_{2}\left(a_{1}^{2}-b_{1}^{2}\right)+2 a_{1} b_{1} b_{2}+2 \frac{a_{1} a_{3}\left(a_{1}^{2}-3 b_{1}^{2}\right)-b_{1} b_{3}\left(b_{1}^{2}-3 a_{1}^{2}\right)}{\sqrt{a_{1}^{2}+b_{1}^{2}}}\right]>0
\end{gathered}
$$




$$
\begin{aligned}
0 & <a_{1}^{2}+b_{1}^{2}+\frac{4}{3} \frac{a_{2}\left(a_{1}^{2}-b_{1}^{2}\right)+2 a_{1} b_{1} b_{2}}{\sqrt{a_{1}^{2}+b_{1}^{2}}}+\frac{23}{15} \frac{a_{1} a_{3}\left(a_{1}^{2}-3 b_{1}^{2}\right)-b_{1} b_{3}\left(b_{1}^{2}-3 a_{1}^{2}\right)}{a_{1}^{2}+b_{1}^{2}} \\
& <\left(k^{2}+\frac{\delta^{2}}{4(1-\lambda)}\right) \sqrt{\frac{a_{2}\left(a_{1}^{2}-b_{1}^{2}\right)+2 a_{1} b_{1} b_{2}+2 \frac{a_{1} a_{3}\left(a_{1}^{2}-3 b_{1}^{2}\right)-b_{1} b_{3}\left(b_{1}^{2}-3 a_{1}^{2}\right)}{\sqrt{a_{1}^{2}+b_{1}^{2}}}}{\frac{3}{2}\left(d_{1}+v\right)}}
\end{aligned}
$$

The solution to the perturbed highly dispersive TSCGE is

$$
\begin{aligned}
q(x, t)= & 4 \arctan \left\{\operatorname { e x p } \left[ \pm\left\{\left(a_{1}^{2}+b_{1}^{2}\right)^{2}+\frac{4}{3}\left[a_{2}\left(a_{1}^{2}-b_{1}^{2}\right)+2 a_{1} b_{1} b_{2}\right] \sqrt{a_{1}^{2}+b_{1}^{2}}\right.\right.\right. \\
& \left.+\frac{23}{15}\left[a_{1} a_{3}\left(a_{1}^{2}-3 b_{1}^{2}\right)-b_{1} b_{3}\left(b_{1}^{2}-3 a_{1}^{2}\right)\right]\right\}^{\frac{1}{2}} \\
& \left.\left.\times \frac{\left(x-x_{0}-v t\right)}{\left(a_{1}^{2}+b_{1}^{2}\right)^{\frac{3}{4}} \sqrt{k^{2}-\delta v-(1-\lambda) v^{2}}}\right]\right\}
\end{aligned}
$$

\section{Conclusions}

This paper has studied the exact solutions to SG type equations, including single, double, and triple sine- and sine-cosine-Gordon equations. These equations included the standard variation, fourth-order dispersion, and sixth-order dispersion. The solutions found are of the topological soliton type, called kinks. After finding the solutions to each unperturbed equation, exact solutions were found for the strongly perturbed variations of each equation. These results will aid in the studies of Josephson junctions, crystal dislocations, ultra-short optical pulses, relativistic field theory, and elementary particles.

Future work should include the use of numerical methods to further study these solutions and possibly find approximate solutions to these equations and other equations of this type. Multiple scale analysis should also be applied to study the effects of weak perturbations on these equations.

\section{Acknowledgements}

This research work was supported by the NSF award \#1901397.

\section{Conflicts of Interest}

The authors declare that they have no conflict of interest.

\section{References}

[1] Barone, A., Esposito, F., Magee, C.J. and Scott, A.C. (1971) Theory and Applications of the Sine-Gordon Equation. La Rivista del Nuovo Cimento, 1, 227-267. https://doi.org/10.1007/BF02820622

[2] Granata, C. and Vettoliere, A. (2016) Nano Superconducting Quantum Interference Device: A Powerful Tool for Nanoscale Investigations. Physics Reports, 614, 1-69. 
https://doi.org/10.1016/j.physrep.2015.12.001

[3] Gubser, D.U. (1998) Navy Programs in Superconducting Technology. Journal of Superconductivity, 11, 1-4.

[4] Fitzgerald, S.P. (2016) Kink Pair Production and Dislocation Motion. Scientific Reports, 6, Article No. 39708. https://doi.org/10.1038/srep39708

[5] Rethfeld, B., Ivanov, D.S., Garcia, M.E. and Anisimov, S.I. (2017) Modeling Ultrafast Laser Ablation. Journal of Physics D: Applied Physics, 50, Article ID: 193001. https://doi.org/10.1088/1361-6463/50/19/193001

[6] Smith, P.W. and Weiner, A.M. (1988) Ultrashort Light Pulses. IEEE Circuits and Devices Magazine, 4, 3-7. https://doi.org/10.1109/101.942

[7] Rajantie, A. (2012) Magnetic Monopoles in Field Theory and Cosmology. Philosophical Transactions of the Royal Society A: Mathematical, Physical, and Engineering Sciences, 370, 5705-5717. https://doi.org/10.1098/rsta.2011.0394

[8] Conti, R., Negro, S. and Tateo, R. (2019) The TT Perturbation and Its Geometric Interpretation. Journal of High Energy Physics, 2019, 85. https://doi.org/10.1007/JHEP02(2019)085

[9] García-Ñustes, M.A., Marín, J.F. and González, J.A. (2017) Bubblelike Structures Generated by Activation of Internal Shape Modes in Two-Dimensional Sine-Gordon Line Solitons. Physical Review E, 95, Article ID: 032222. https://doi.org/10.1103/PhysRevE.95.032222

[10] Kowalczyk, M., Martel, Y. and Muñoz, C. (2017) Kink Dynamics in the $\phi^{4}$ Model: Asymptotic Stability for Odd Perturbations in the Energy Space. Journal of the American Mathematical Society, 30, 769-798. https://doi.org/10.1090/jams/870

[11] Arthur, R., Dorey, P. and Parini, R. (2016) Breaking Integrability at the Boundary: The Sine-Gordon Model with Robin Boundary Conditions. Journal of Physics A: Mathematical and Theoretical, 49, Article ID: 165205. https://doi.org/10.1088/1751-8113/49/16/165205

[12] Gharaati, A., Fathi, N., Johnson, S. and Biswas, A. (2012) Analytic Solutions of Fluxons in a Non-Homogeneous Josephson Junction. Waves in Random and Complex Media, 22, 249-259. https://doi.org/10.1080/17455030.2011.652365

[13] Fabian, A., Kohl, R. and Biswas, A. (2009) Perturbation of Topological Solitons Due to Sine-Gordon Equation and Its Type. Communications in Nonlinear Science and Numerical Simulation, 14, 1227-1244. https://doi.org/10.1016/j.cnsns.2008.01.013

[14] Ebadi, G., Johnson, S., Zerrad, E. and Biswas, A. (2012) Solitons and Other Nonlinear Waves for the Perturbed Boussinesq Equation with Power Law Nonlinearity. Journal of King Saud University, 24, 237-241. https://doi.org/10.1016/j.jksus.2011.05.001

[15] Ebadi, G., Mojaver, A., Johnson, S., Kumar, S. and Biswas, A. (2012) Dynamics of Dispersive Topological Solitons and Its Perturbations. Indian Journal of Physics, 86, 1115-1129. https://doi.org/10.1007/s12648-012-0172-5

[16] Johnson, S. and Biswas, A. (2011) Perturbation of Dispersive Topological Solitons. Physica Scripta, 84, Article ID: 015002. https://doi.org/10.1088/0031-8949/84/01/015002

[17] Bogdan, M.M., Kosevich, A.M. and Maugin, G.A. (2001) Soliton-Complex Dynamics in Strongly Dispersive Medium. Wave Motion, 34, 1-26. https://doi.org/10.1016/S0165-2125(01)00066-X

[18] Kivshar, Y. and Malomed, B. (1989) Dynamics of Solitons in Nearly Integrable Systems. Reviews of Modern Physics, 61, 763-915. 
https://doi.org/10.1103/RevModPhys.61.763

[19] Chirilus-Bruckner, M., Chong, C., Cuevas-Maraver, J. and Kevrekidis, P.G. (2014) Sine-Gordon Equation: From Discrete to Continuum. Nonlinear Systems and Complexity, 10, 31-57. https://doi.org/10.1007/978-3-319-06722-3 2 